\title{
REVIEW
}

\section{Year in review 2010: Critical Care - multiple organ dysfunction and sepsis}

\author{
Etienne de Montmollin and Djillali Annane*
}

\begin{abstract}
This review summarizes new insights in the pathophysiologic implications of inflammation and microvascular alterations in organ dysfunction, as well as genetic factor contribution, from clinical and experimental studies that were published in 2010 in Critical Care in the fields of multiple organ dysfunction and sepsis. New diagnostic and prognostic markers of organ dysfunction are presented. Evaluations of novel therapeutic strategies, including implementation of international guidelines, modulation of inflammation and coagulation, and prevention of ventilator-induced lung injury and acute kidney injury, are reported. The results of these experimental studies and clinical trials are discussed in the context of the current relevant scientific and clinical background.
\end{abstract}

\section{Introduction}

Multiple organ failure, or multiple organ dysfunction syndrome (MODS), was defined by the 1991 Consensus Conference of the American College of Chest Physicians and the Society of Critical Care Medicine as 'the presence of altered organ functions in an acutely ill patient such that homeostasis cannot be maintained without intervention' [1]. Septic shock is the main cause of MODS in intensive care units, and the intensity of MODS is correlated directly to mortality [2]. Furthermore, MODS is the main cause of death in patients with severe sepsis, representing $43.1 \%$ of patients in a recent retrospective study [3]. Our aim was to review the relevant findings of research articles that were published in 2010 in Critical Care and that focused on advances in the understanding of MODS physiopathology, diagnostic and prognostic marker evaluation, and novel therapy strategies.

*Correspondence: djillali.annane@rpc.aphp.fr

Service de Réanimation Polyvalente, Hôpital Raymond Poincaré (AP-HP), Université de Versailles Saint Quentin, 104, bd. Raymond Poincaré, 92380 Garches, France

\section{Inflammation, septic microvascular dysfunction, and multiple organ dysfunction syndrome}

It is now well established that the correction of macrovascular hemodynamic parameters is not sufficient to prevent MODS in sepsis and that persistent microvascular alteration is associated with the development of organ dysfunction and death [4]. The endothelium plays a central role in microvascular dysfunction and sepsis physiopathology, regulating vasomotor tone, cellular trafficking, coagulation, and local balance between proand anti-inflammatory mediators [5].

In a prospective single-center study of 221 patients admitted with a clinical suspicion of infection to an emergency department, Shapiro and colleagues [6] investigated the association between endothelial cell signaling activation during sepsis at the time of emergency department consultation and the subsequent severity of organ dysfunction. They demonstrated that circulating levels of biomarkers of endothelial activation such as soluble fms-like tyrosine kinase-1 (sFlt-1), plasminogen activator inhibitor-1 (PAI-1), soluble Eselectin, soluble intercellular adhesion molecule-1 (sICAM-1), and soluble vascular cell adhesion molecule-1 (sVCAM-1) were strongly correlated with sepsis severity. Among those biomarkers, sFlt-1, which is the soluble form of the vascular endothelial growth factor-1 (VEGF-1) receptor, had the strongest association with Sequential Organ Failure Assessment (SOFA) score. sFlt-1 contributes to endothelial cell activation and correlates with inflammatory cascade activation [7], making the VEGF signaling axis a potential target for endothelial activation modulation.

Activation of the renin-angiotensin system (RAS) affects microvascular function through promotion of leukostasis and induction of capillary permeability. The RAS also promotes oxidative stress and endothelial dysfunction after lipopolysaccharide injection in mice, a phenomenon corrected by an angiotensin-converting enzyme inhibitor or an angiotensin receptor blocker [8]. Doerschug and colleagues [9] demonstrated that RAS activation also correlates with microvascular dysfunction and organ failure in humans. In a prospective singlecenter study of 30 patients with severe sepsis, the authors 
showed that RAS mediators (plasma renin activity and angiotensin II) were increased in clinical sepsis and persisted despite a corrected mean arterial pressure. Furthermore, early angiotensin II levels correlated with the SOFA score at day 1, and RAS mediators correlated negatively with the microvascular hyperemic response to ischemia.

Angiopoietin-2 (Ang-2) is an angiogenic peptide stored predominantly in the Weibel-Palade bodies of endothelial cells, and serum levels are correlated with sepsis mortality and the extent of organ dysfunction [10]. In an observational prospective study of 85 patients with sepsis, Davis and colleagues [11] confirmed that Ang-2 levels were increased in sepsis and correlated with SOFA score. The authors also demonstrated that Ang-2 levels were inversely correlated to endothelial nitric oxide (NO) bioavailability, as measured by reactive hyperemiaperipheral artery tonometry. As $\mathrm{NO}$ is an inhibitor of exocytosis of Weibel-Palade bodies, the authors suggested that low endothelial NO bioavailability is at least partly responsible for the increased levels of Ang-2 in sepsis, which induce endothelial activation and microvascular dysfunction.

Endothelial cell activation in sepsis is a key factor of microvascular dysfunction leading to MODS. Lectin-like oxidized low-density lipoprotein receptor-1 (LOX-1) is an endothelial cell membrane protein that recognizes oxidized low-density lipoproteins, damaged or apoptotic cells, endotoxins, and pathogenic microorganisms [12]. LOX-1 is a potent mediator of endothelial dysfunction, induces superoxide generation, and enhances endothelial adhesiveness to leukocytes and chemokine production. In an experimental study of endotoxemic rats, Landsberger and colleagues [13] evaluated the effects of the administration of anti-LOX-1 by antibodies on intestinal capillary perfusion and leukocyte adhesion by using intravital microscopy. The authors showed that LOX-1 neutralization reduced endotoxin-induced leukocyte adherence and reduced plasma levels of monocyte chemoattractant protein-1 (MCP-1), a major chemokine. Hence, LOX-1 represents a novel target for the modulation of inflammation within the microcirculation in sepsis.

Septic shock is characterized by a relative deficiency in vasopressin, and exogenous administration of arginine vasopressin (AVP) restores vasomotor tone and blood pressure. Thus, AVP is recommended by the Surviving Sepsis Campaign (SSC) as an adjunctive therapy in refractory septic shock. However, the Vasopressin in Septic Shock Trial (VASST), a large randomized controlled trial (RCT) that compared AVP with norepinephrine for the treatment of septic shock, did not find any benefit on mortality [14]. AVP is a mixed agonist of V1a receptor and $\mathrm{V} 2$ receptor $(\mathrm{V} 2 \mathrm{R})$, and there is increasing evidence that endothelial V2Rs may aggravate sepsis-induced vasodilatation, fluid accumulation, leukocyte rolling, and microvascular thrombosis. Hence, Rehberg and colleagues [15] evaluated the effects of a selective V2R antagonist in an experimental septic model of ovine fecal peritonitis. Infusion of a V2R antagonist, compared with placebo and AVP, improved survival, mean arterial pressure, and cardiac index as well as metabolic, liver, and renal dysfunction. Along with AVP, selective V2R antagonists and V1a receptor agonists represent promising new therapies.

Since the publication of the first SSC international guidelines in 2004 [16], the main objective of the intensive care community has been the implementation of such guidelines through intervention bundles. Cardoso and colleagues [17] evaluated the implementation of a core 6-hour bundle in a network of Portuguese ICUs. Between December 2004 and November 2005, the authors prospectively evaluated the level of compliance with SSC guideline recommendations and its incidence on mortality in all patients admitted with communityacquired sepsis to 17 ICUs that represented $41 \%$ of all Portuguese ICU beds. The core 6-hour bundle consisted of serum lactate measurement, crystalloid or colloid infusion, blood cultures and other specimens for microbiology before antibiotherapy, antibiotherapy administered within the first hour of diagnosis, and vasopressor administration if the mean arterial pressure was less than $65 \mathrm{~mm} \mathrm{Hg}$. The authors showed that - after adjustment for sepsis severity, Simplified Acute Physiology Score II (SAPS II), and number of comorbidities - the two actions significantly associated with an improvement in 28-day mortality were sampling for blood cultures (odds ratio (OR) 0.53) and vasopressor administration (OR 0.51). Furthermore, the full completion of the six actions of the bundle significantly decreased 28-day mortality (OR $0.44)$, and the number of patients to treat to save one life was 6. However, compliance was poor; only $12 \%$ of patients with severe sepsis completed all six actions of the bundle. These results confirmed the clinical relevance of care bundle implementation and should prompt actions to improve compliance with such bundles.

\section{Septic myocardial dysfunction}

Cardiac diastolic dysfunction is frequent in septic shock. Tissue Doppler imaging (TDI) is a recent echocardiographic technique that measures myocardial velocities of the mitral annulus. Peak early diastolic transmitral/peak early diastolic annular velocity $\left(E / e^{\prime}\right)$, in particular, has been validated for evaluation of diastolic dysfunction, and a study by Mousavi and colleagues [18] in 2010 confirmed its feasibility in the intensive care setting. In a study of 21 patients with septic shock, Sturgess and colleagues [19] sought to prospectively evaluate the prognostic significance of TDI variables in comparison 
with cardiac biomarkers troponin T, B-type natriuretic peptide (BNP), and $\mathrm{N}$-terminal pro-B-type natriuretic peptide (NT-proBNP). Systolic dysfunction was present in $67 \%$ of patients, and diastolic dysfunction was present in $57 \%$ of patients. The authors showed that, for hospital mortality, E/e' at a threshold value of 14.5 offered 100\% sensitivity and $83 \%$ specificity and was an independent predictor of hospital mortality. When area under the receiver operating characteristic curves were compared, E/e' offered better prognostic prediction of hospital mortality in septic shock than cardiac biomarkers troponin T, BNP, and NT-proBNP. Thus, echographic techniques evaluating diastolic dysfunction in sepsis may replace biomarkers for mortality prediction.

\section{Sepsis and acute kidney injury}

Acute kidney injury (AKI) is a frequent complication in sepsis; an incidence of $20 \%$ and an associated mortality of up to $35 \%$ have been reported [20]. In this context, diagnostic and prognostic markers of AKI, such as cystatin C and serum neutrophil gelatinase-associated lipocalin (NGAL), could improve management of such patients.

In a study of 444 ICU patients, Nejat and colleagues [21] prospectively studied the diagnostic and predictive performance of urinary cystatin $\mathrm{C}(\mathrm{uCysC})$ for $\mathrm{AKI}$ and 30 -day ICU mortality. AKI was defined as a $50 \%$ or $0.3 \mathrm{mg} / \mathrm{dL}$ increase in plasma creatinine above baseline level. Eighteen percent of the patients had sepsis, $45 \%$ had AKI, and ICU mortality was $14 \%$. The authors found that $\mathrm{uCysC}$ measured at ICU admission was independently associated with sepsis (OR 3.43), AKI (OR 1.49), and mortality (OR 1.6) and that $\mathrm{uCysC}$ concentrations were significantly higher in the presence of sepsis or AKI. $\mathrm{uCysC}$ was predictive of sepsis with an optimal cut-point of $0.24 \mathrm{mg} / \mathrm{L}$. In the sepsis group, however, diagnostic performance of uCysC for AKI was not significant but was predictive of AKI within 48 hours. On the other hand, plasmatic cystatin $\mathrm{C}$ was not independently associated with sepsis or mortality but was predictive of AKI. Thus, uCysC seems to be a more promising biomarker than plasmatic cystatin $\mathrm{C}$, and prediction of $\mathrm{AKI}$ and mortality is of important clinical relevance.

Serum or urinary NGAL is another extensively studied biomarker of AKI and has shown predictive value for AKI, AKI severity, and AKI-related outcomes such as need for renal replacement therapy (RRT) and mortality [22]. Kümpers and colleagues [23] prospectively evaluated the prognostic utility of serum NGAL at inception of RRT in 109 critically ill patients with established AKI, of whom $51.4 \%$ had sepsis. The authors showed that serum NGAL concentrations were significantly higher in patients with sepsis and increased across patient groups when stratified by the renal variable of the SOFA score. NGAL level was higher in non-survivors compared with survivors and was identified as an independent predictor of 28-day mortality. At RRT initiation, an optimal cutoff value of greater than $360 \mathrm{ng} / \mathrm{mL}$ had a sensitivity of $82.1 \%$ and a specificity of $59.2 \%$ for 28 -day mortality. However, serum NGAL levels were not associated with renal recovery, ventilation-free days, or ICU-free days at 28 days.

Fluid resuscitation is a major therapeutic goal in critically ill patients with sepsis. However, there is much controversy about the optimal fluid solution to use. Colloids - hydroxyethly starch (HES), in particular - are suspected to induce or aggravate AKI. An international prospective cohort study that was published in 2008 and that included 1,013 patients in shock found that the use of artificial hyperoncotic colloids or hyperoncotic albumin was associated with an increased risk of renal function deterioration or need for dialysis [24]. Furthermore, the use of hyperoncotic albumin was associated with an increased risk of ICU death. In this setting, Wiedermann and colleagues [25] conducted a metaanalysis of RCTs that evaluated AKI after infusion of hyperoncotic albumin and hyperoncotic HES solutions. Eleven RCTs, representing a total of 1,220 patients, were included in the analysis. Hyperoncotic albumin decreased the odds of AKI by 76\%, whereas hyperoncotic HES showed the opposite effect, increasing the odds of AKI by $92 \%$. Furthermore, hyperoncotic albumin decreased the odds of mortality by $48 \%$, whereas hyperoncotic HES raised the odds of mortality by $92 \%$. The authors concluded that hyperoncotic fluids per se do not appear to induce AKI, given that the renal risk is linked to the type of colloid used. To clarify this controversy, the CRISTAL trial (Colloids Compared to Crystalloids in Fluid Resuscitation of Critically Ill Patients: A Multinational Randomised Controlled Trial), a large ongoing RCT, is evaluating the efficacy and safety of any type of colloids versus crystalloids for fluid resuscitation in critically ill patients (clinical trials number NCT00318942).

\section{Acute lung injury and acute respiratory distress syndrome}

Ventilator-induced lung injury (VILI) induces oxidative stress and reactive oxygen species (ROS) such as peroxynitrites [26]. Oxidative DNA damage induces activation of the repair nuclear enzyme poly(ADP-ribose) polymerase (PARP), whose overactivation is detrimental to the cell by depleting ATP stores. The peroxynitrite-PARP pathway is thought to play a role in septic endothelial dysfunction and multiple organ failure and particularly in acute renal failure caused by mechanical ventilation in the course of sepsis. In a mouse model of sepsis, Vaschetto and colleagues [27] demonstrated that high tidal ventilation activated the peroxynitrite-PARP pathway and that a peroxynitrite decomposition catalyst or a 
PARP inhibitor attenuated renal endothelial dysfunction and improved renal perfusion and renal function. This work confirmed that the peroxynitrite-PARP pathway is an interesting therapeutic target for the management of VILI and VILI-induced organ failure.

\section{Coagulation and multiple organ dysfunction syndrome}

Outcome in severe sepsis and septic shock is at least partly conditioned by predisposing factors such as genetic susceptibility and response to infection. Activation of inflammation and coagulation in sepsis leads to an imbalance between fibrin formation and fibrinolysis. PAI-1 is a key protein for fibrinolysis inhibition and its overexpression contributes to multiple organ failure [28]. In this context, Madách and colleagues [29] aimed to prospectively evaluate 207 Caucasian patients with pneumonia-induced severe sepsis for the effects of the 4G/5G and 4G/4G polymorphisms of the PAI-1 gene, in which the $4 \mathrm{G}$ allele results in greater transcription of the gene. The authors showed that carriers of the two polymorphisms, compared with those of the 5G/5G polymorphism, had higher disseminated intravascular coagulation scores and had a 2.74-fold higher risk of MODS and a 2.57 -fold higher risk of septic shock. However, 4G/5G and 4G/4G polymorphisms were not associated with a difference in mortality in comparison with 5G/5G patients, suggesting that a single polymorphism cannot account for the overall septic shock morbimortality. Furthermore, those results require validation in an independent cohort.

Recombinant human activated protein C (rhAPC) is an anticoagulant protein that downregulates inflammation and apoptosis. It has shown significant survival benefit in patients with severe sepsis [30]. However, doubts about the protective effects of rhAPC in sepsis persist. Thus, Maybauer and colleagues [31] undertook an experimental study evaluating cardiovascular and microcirculatory effects of rhAPC in a sheep model of acute lung injury and septic shock. The two-hit septic animal model consisted of sheep subjected to smoke inhalation followed by lung instillation of live Pseudomonas aeruginosa. In these sheep, in comparison with controls, infusion of rhAPC significantly improved cardiovascular function with maintained mean arterial pressure and cardiac index. Furthermore, rhAPC attenuated changes in visceral and cerebral microcirculation. Heart tissue analysis revealed reductions of 3-nitrotyrosine and malondialdehyde, which are, respectively, markers of tissue injury caused by ROS and an indicator of lipid peroxydation and oxidative stress. Those experimental findings provide evidence of a beneficial effect of rhAPC in septic shock. However, a recent meta-analysis of RCTs that assessed the effects of rhAPC for severe sepsis in adults and children found no evidence of 28-day mortality improvement and a higher risk of bleeding [32]. In this context of conflicting results, two large randomized trials are under way: the PROWESS-SHOCK (Protein C Worldwide Evaluation in Severe Sepsis-Shock ) trial is evaluating the efficacy and safety of drotrecogin alfa in adult patients with septic shock (clinical trials number NCT00604214), and the APROCCHS (Activated Protein C and Corticosteroids for Human Septic Shock) study is evaluating the efficacy and interactions of rhAPC and corticosteroids in septic shock (clinical trials number NCT00625209).

\section{Metabolic dysfunction}

Because it has shown improvement of mortality in critically ill patients, tight glycemic control is a supportive therapy validated in the SSC guidelines [33]. However, a more recent $\mathrm{RCT}$ evaluating intensive versus conventional glucose control in critically ill patients showed conflicting results, and tight glycemic control resulted in increased mortality [34]. Chase and colleagues [35] prospectively evaluated (before-after study) a novel tight glycemic control protocol called SPRINT (Specialized Relative Insulin and Nutrition Titration), which is based on control of both insulin and nutrition inputs. SPRINT modulates nutritional intakes and insulin on the basis of response to the prior insulin and nutrition interventions and estimation of insulin sensitivity of the patient. The authors previously reported that the SPRINT protocol reduced hospital mortality by $25 \%$ to $40 \%$ for patients staying 3 to 5 days in the ICU [35]. In a study published in Critical Care in 2010, the same team evaluated the impact of the SPRINT protocol on organ failure [36]. In that retrospective cohort study, the team compared evolution of SOFA scores in 413 pre-SRINT critically ill patients versus 371 SPRINT patients. The authors showed that SPRINT tight glycemic control reduced total organ failures and increased organ failure-free days, which is the presumed causative factor for reduced mortality in SPRINT patients. The SPRINT protocol brings a new and interesting approach to tight glycemic control and warrants further evaluation in a randomized controlled study.

\section{Septic cerebral dysfunction}

The pathophysiology of brain dysfunction, among the various organ dysfunctions, remains poorly understood. Septic encephalopathy is a complex phenomenon involving alteration of the blood-brain barrier, local inflammation, activation of the complement cascade, excessive leukocyte recruitment, local NO production, and alteration of neurotransmitter systems [37]. Taccone and colleagues [38] demonstrated that, despite maintained mean arterial pressure, cerebral microvascular impairment occurred in sepsis. In an animal model of sheep 
peritonitis, the authors used the sidestream dark field videomicroscopy technique to show that sepsis induced decreases in cerebral total perfused vessel density, functional capillary density, the proportion of small perfused vessels, and the number of perfused capillaries [38].

\section{Abbreviations}

AKl, acute kidney injury; Ang-2, angiopoietin-2; AVP, arginine vasopressin BNP, B-type natriuretic peptide; E/e', peak early diastolic transmitral/peak early diastolic annular velocity; HES, hydroxyethly starch; ICU, intensive care unit; LOX-1, lectin-like oxidized low-density lipoprotein receptor-1; MODS, multiple organ dysfunction syndrome; NGAL, neutrophil gelatinaseassociated lipocalin; NO, nitric oxide; NT-proBNP, N-terminal pro-B-type natriuretic peptide; OR, odds ratio; $\mathrm{PAl}-1$, plasminogen activator inhibitor-1; PARP, poly(ADP-ribose) polymerase; RAS, renin-angiotensin system; RCT, randomized controlled trial; rhAPC, recombinant human activated protein C; ROS, reactive oxygen species; RRT, renal replacement therapy; sFlt-1, soluble fms-like tyrosine kinase-1; SOFA, Sequential Organ Failure Assessment; SPRINT, Specialized Relative Insulin and Nutrition Titration; SSC, Surviving Sepsis Campaign; TDI, tissue Doppler imaging; UCysC, urinary cystatin C; VEGF, vascular endothelial growth factor; VILI, ventilator-induced lung injury; V2R, V2 receptor.

\section{Competing interests}

The authors declare that they have no competing interests.

Published: 5 December 2011

\section{References}

1. American College of Chest Physicians/Society of Critical Care Medicine Consensus Conference: definitions for sepsis and organ failure and guidelines for the use of innovative therapies in sepsis. Crit Care Med 1992, 20:864-874.

2. Blanco J, Muriel-Bombín A, Sagredo V, Taboada F, Gandía F, Tamayo L, Collado J, García-Labattut A, Carriedo D, Valledor M, De Frutos M, López MJ, Caballero A, Guerra J, Alvarez B, Mayo A, Villar J; Grupo de Estudios y Análisis en Cuidados Intensivos: Incidence, organ dysfunction and mortality in severe sepsis: a Spanish multicentre study. Crit Care 2008, 12:R158.

3. Vincent JL, Nelson DR, Williams MD: Is worsening multiple organ failure the cause of death in patients with severe sepsis? Crit Care Med 2011, 39:1050-1055.

4. Chierego M, Verdant C, De Backer D: Microcirculatory alterations in critically ill patients. Minerva Anestesiol 2006, 72:199-205.

5. Aird WC: The role of the endothelium in severe sepsis and multiple organ dysfunction syndrome. Blood 2003, 101:3765-3777.

6. Shapiro N, Schuetz P, Yano K, Sorasaki M, Parikh S, Jones A, Trzeciak S, Ngo L, Aird W: The association of endothelial cell signaling, severity of illness, and organ dysfunction in sepsis. Crit Care 2010, 14:R182.

7. Shapiro NI, Yano K, Okada H, Fischer C, Howell M, Spokes KC, Ngo L, Angus DC, Aird WC: A prospective, observational study of soluble FLT-1 and vascular endothelial growth factor in sepsis. Shock 2008, 29:452-457.

8. Lund DD, Brooks RM, Faraci FM, Heistad DD: Role of angiotensin II in endothelial dysfunction induced by lipopolysaccharide in mice. Am J Physiol Heart Circ Physiol 2007, 293:H3726-3731.

9. Doerschug K, Delsing A, Schmidt G, Ashare A: Renin-angiotensin system activation correlates with microvascular dysfunction in a prospective cohort study of clinical sepsis. Crit Care 2010, 14:R24.

10. Siner JM, Bhandari V, Engle KM, Elias JA, Siegel MD: Elevated serum angiopoietin 2 levels are associated with increased mortality in sepsis. Shock 2009, 31:348-353.

11. Davis J, Yeo T, Piera K, Woodberry T, Celermajer D, Stephens D, Anstey N: Angiopoietin-2 is increased in sepsis and inversely associated with nitric oxide-dependent microvascular reactivity. Crit Care 2010, 14:R89.

12. Chen M, Masaki T, Sawamura T: LOX-1, the receptor for oxidized low-density lipoprotein identified from endothelial cells: implications in endothelial dysfunction and atherosclerosis. Pharmacol Ther 2002, 95:89-100.

13. Landsberger M, Zhou J, Wilk S, Thaumüller C, Pavlovic D, Otto M, Whynot S, Hung O, Murphy MF, Cerny V, Felix SB, Lehmann C: Inhibition of lectin-like oxidized low-density lipoprotein receptor-1 reduces leukocyte adhesion within the intestinal microcirculation in experimental endotoxemia in rats.
Crit Care 2010, 14:R223

14. Russell JA, Walley KR, Singer J, Gordon AC, Hébert PC, Cooper DJ, Holmes CL, Mehta S, Granton JT, Storms MM, Cook DJ, Presneill JJ, Ayers D; VASST Investigators: Vasopressin versus norepinephrine infusion in patients with septic shock. N Engl J Med 2008, 358:877-887.

15. Rehberg S, Ertmer C, Lange M, Morelli A, Whorton E, Dünser M, Strohhäcker AK, Lipke E, Kampmeier TG, Van Aken H, Traber DL, Westphal M: Role of selective V(2)-receptor-antagonism in septic shock: a randomized, controlled, experimental study. Crit Care 2010, 14:R200.

16. Dellinger RP, Carlet JM, Masur H, Gerlach H, Calandra T, Cohen J, GeaBanacloche J, Keh D, Marshall JC, Parker MM, Ramsay G, Zimmerman JL, Vincent JL, Levy MM; Surviving Sepsis Campaign Management Guidelines Committee: Surviving Sepsis Campaign guidelines for management of severe sepsis and septic shock. Crit Care Med 2004, 32:858-873.

17. Cardoso T, Carneiro AH, Ribeiro O, Teixeira-Pinto A, Costa-Pereira A: Reducing mortality in severe sepsis with the implementation of a core 6-hour bundle: results from the Portuguese community-acquired sepsis study (SACiUCI study). Crit Care 2010, 14:R83.

18. Mousavi N, Czarnecki A, Ahmadie R, Tielan F, Kumar K, Lytwyn M, Kumar A, Jassal DS: The utility of tissue Doppler imaging for the noninvasive determination of left ventricular filling pressures in patients with septic shock. J Intensive Care Med 2010, 25:163-167.

19. Sturgess D, Marwick T, Joyce C, Jenkins C, Jones M, Masci P, Stewart D, Venkatesh B: Prediction of hospital outcome in septic shock: a prospective comparison of tissue Doppler and cardiac biomarkers. Crit Care 2010, 14:R44.

20. Schrier RW, Wang W: Acute renal failure and sepsis. N Engl J Med 2004, 351:159-169.

21. Nejat M, Pickering J, Walker R, Westhuyzen J, Shaw G, Frampton C, Endre Z: Urinary cystatin $C$ is diagnostic of acute kidney injury and sepsis, and predicts mortality in the intensive care unit. Crit Care 2010, 14:R85.

22. Wheeler DS, Devarajan P, Ma Q, Harmon K, Monaco M, Cvijanovich N, Wong HR: Serum neutrophil gelatinase-associated lipocalin (NGAL) as a marker of acute kidney injury in critically ill children with septic shock. Crit Care Med 2008, 36:1297-1303.

23. Kümpers P, Hafer C, Lukasz A, Lichtinghagen R, Brand K, Fliser D, Faulhaber Walter R, Kielstein J: Serum neutrophil gelatinase-associated lipocalin at inception of renal replacement therapy predicts survival in critically ill patients with acute kidney injury. Crit Care 2010, 14:R9.

24. Schortgen F, Girou E, Deye N, Brochard L: The risk associated with hyperoncotic colloids in patients with shock. Intensive Care Med 2008, 34:2157-2168

25. Wiedermann C, Dunzendorfer S, Gaioni L, Zaraca F, Joannidis M: Hyperoncotic colloids and acute kidney injury: a meta-analysis of randomized trials. Crit Care 2010, 14:R191.

26. Martínez-Caro L, Lorente JA, Marín-Corral J, Sánchez-Rodríguez C, SánchezFerrer A, Nin N, Ferruelo A, de Paula M, Fernández-Segoviano P, Barreiro E, Esteban $A$ : Role of free radicals in vascular dysfunction induced by high tidal volume ventilation. Intensive Care Med 2009, 35:1110-1119.

27. Vaschetto R, Kuiper J, Musters R, Eringa E, Della Corte F, Murthy K, Groeneveld AJ, Plötz F: Renal hypoperfusion and impaired endothelium-dependent vasodilation in an animal model of VILI: the role of the peroxynitrite-PARP pathway. Crit Care 2010, 14:R45.

28. Kornelisse RF, Hazelzet JA, Savelkoul HF, Hop WC, Suur MH, Borsboom AN, Risseeuw-Appel IM, van der Voort E, de Groot R: The relationship between plasminogen activator inhibitor-1 and proinflammatory and counterinflammatory mediators in children with meningococcal septic shock. J Infect Dis 1996, 173:1148-1156.

29. Madách K, Aladzsity I, Szilágyi Á, Fust G, Gál J, Pénzes I, Prohászka Z: 4G/5G polymorphism of PAl-1 gene is associated with multiple organ dysfunction and septic shock in pneumonia induced severe sepsis: prospective, observational, genetic study. Crit Care 2010, 14:R79.

30. Bernard GR, Vincent JL, Laterre PF, LaRosa SP, Dhainaut JF, Lopez-Rodriguez A Steingrub JS, Garber GE, Helterbrand JD, Ely EW, Fisher CJ Jr.; Recombinant human protein C Worldwide Evaluation in Severe Sepsis (PROWESS) study group: Efficacy and safety of recombinant human activated protein $\mathrm{C}$ for severe sepsis. N Engl J Med 2001, 344:699-709.

31. Maybauer MO, Maybauer DM, Fraser JF, Szabo C, Westphal M, Kiss L, Horvath EM, Nakano Y, Herndon DN, Traber LD, Traber DL: Recombinant human activated protein $C$ attenuates cardiovascular and microcirculatory dysfunction in acute lung injury and septic shock. Crit Care 2010, 14:R217. 
32. Marti-Carvajal AJ, Sola I, Lathyris D, Cardona AF: Human recombinant activated protein C for severe sepsis. Cochrane Database Syst Rev 2011, 4:CD004388.

33. van den Berghe $G$, Wouters $P$, Weekers F, Verwaest $C$, Bruyninckx F, Schetz M Vlasselaers D, Ferdinande P, Lauwers P, Bouillon R: Intensive insulin therapy in the critically ill patients. N Engl J Med 2001, 345:1359-1367.

34. NICE-SUGAR Study Investigators, Finfer S, Chittock DR, Su SY, Blair D, Foster D, Dhingra V, Bellomo R, Cook D, Dodek P, Henderson WR, Hébert PC, Heritier S, Heyland DK, McArthur C, McDonald E, Mitchell I, Myburgh JA, Norton R, Potter J, Robinson BG, Ronco JJ: Intensive versus conventional glucose control in critically ill patients. N Engl J Med 2009, 360:1283-1297.

35. Chase JG, Shaw G, Le Compte A, Lonergan T, Willacy M, Wong XW, Lin J, Lotz T, Lee D, Hann C: Implementation and evaluation of the SPRINT protocol for tight glycaemic control in critically ill patients: a clinical practice change. Crit Care 2008, 12:R49.
36. Chase JG, Pretty CG, Pfeifer L, Shaw GM, Preiser JC, Le Compte AJ, Lin J, Hewett D, Moorhead KT, Desaive T: Organ failure and tight glycemic control in the SPRINT study. Crit Care 2010, 14:R154.

37. Jacob A, Brorson JR, Alexander JJ: Septic encephalopathy: inflammation in man and mouse. Neurochem Int 2011, 58:472-476.

38. Taccone FS, Su F, Pierrakos C, He X, James S, Dewitte O, Vincent J-L, De Backer $D$ : Cerebral microcirculation is impaired during sepsis: an experimental study. Crit Care 2010, 14:R140.

doi:10.1186/cc10359

Cite this article as: de Montmollin E, Annane D: Year in review 2010: Critical Care - multiple organ dysfunction and sepsis. Critical Care 2011, 15:236. 